\title{
Use of a Novel Fiber Optical Strain Sensor for Monitoring the Vertical Deflection of an Aircraft Flap
}

\author{
Gaizka Durana, Marlene Kirchhof, Michael Luber, Idurre Sáez de Ocáriz, Hans Poisel, Member, IEEE, \\ Joseba Zubia, and Carmen Vázquez
}

\begin{abstract}
The present paper reports the use of a plastic optical fiber-based sensor for elongation measurements in an aircraft flap subjected to different types of flexural loading conditions. The sensor, bonded to the surface of the aircraft structure, relies on measuring the phase shift that occurs between two sinusoidally modulated light signals when the aircraft structure is bent. The light signals are guided through two optical fibers, one of them fixed to the top surface of the flap, and the other one to the bottom surface. The sensor offers good signal stability and repeatability and represents a cost-effective alternative to other more sophisticated health-monitoring systems currently used.
\end{abstract}

Index Terms-Composite structures, elongation, flexural tests, plastic optical fiber (POF), POF-based sensors.

\section{INTRODUCTION}

I N RECENT YEARS, there have been efforts for developing load-bearing structures that include health-monitoring systems. These systems represent an important aspect in the maintenance of different types of structures (e.g., bridges, roofs of sport centers, blades of helicopters or of wind power plants, airplane wings, etc.) through the use of embedded or surfacebonded sensors. In the case of the aerospace industry, the continuous flutter of wings during flight reduces significantly their service life. Therefore, a real-time self-diagnostic system that has the ability to monitor the dynamic response of these structures under the influence of loading conditions is clearly interesting.

Fiber-optic sensors offer many advantages over their electrical counterparts- these include their electromagnetic immunity, light weight and minimal intrusiveness when embedded

Manuscript received January 13, 2009; revised March 09, 2009; accepted April 22, 2009. Current version published August 28, 2009. This work was supported by the Institution Ministerio de Educación y Ciencia, Universidad del País Vasco/Euskal Herriko Unibertsitatea, Gobierno Vasco/Eusko Jaurlaritza, Diputación Foral de Bizkaia/Bizkaiko Foru Aldundia, and the European Union Seventh Research Framework Program under Project TEC2006-13273C03-01, Project GIU05/03, Project EJIE07/12, Project HEGATEK-05, Project SHMSENS, Project S-PE07CA05, and AISHAII, respectively. The associate editor coordinating the review of this paper and approving it for publication was Prof. Bernhard Jakoby.

G. Durana is with the Department of Electronics and Telecommunications, University of the Basque Country, E-48013 Bilbao, Spain (e-mail: gaizka.durana@ehu.es).

M. Kirchhof, M. Luber, and H. Poisel are with the POF Application Center, D-90489 Nuremberg, Germany.

I. S. de Ocáriz is with the Aeronautical Technologies Center CTA, Vitoria, Spain

J. Zubia is with the University of the Basque Country, E-48013 Bilbao, Spain. C. Vázquez is with the Universidad Carlos III, E-28911 Leganés, Madrid, Spain.

Color versions of one or more of the figures in this paper are available online at http://ieeexplore.ieee.org.

Digital Object Identifier 10.1109/JSEN.2009.2029109 in load-bearing structures. These sensors have already been introduced and demonstrated in structural monitoring of several physical quantities, including deflection, vibration, and temperature [1]-[11].

Although a number of approaches, such as fiber Bragg grating technology or interferometric techniques offer excellent performance, they generally require expensive signal processing hardware to acquire and analyze the optical signal [12], [13]. However, the high measurement resolution provided by these sensors is often unnecessary and other simpler and more cost-effective optical fiber-based sensing schemes may offer an interesting alternative for integration of the sensors into structures. The transduction technique based on intensity modulation of light provides simple and potentially low-cost devices because it is easier to measure the optical power than the phase or the state of polarization of light [14], [15]. In addition, this is the most suitable approach with multimode fibers because the phase and state of polarization is lost when light propagates through this kind of fibers.

Recently, plastic optical fibers (POFs) have been attracting a considerable amount of attention due to a number or reasons. Amongst the advantages, their low cost, ease of termination and coupling, and their relatively high resistance to fracture can be mentioned. Furthermore, their use as transducers in sensing applications requires no more than light emitting diodes and photodiodes, and as the sensing principle relies on the modulation of light intensity, sophisticated signal interrogation techniques are not necessary [2], [16]-[18]. For all these reasons, they are more simple to handle in field applications than their glass counterparts.

This paper reports, for the first time, the results of a series of tests in which a POF-based sensor was used to monitor the deflection of an aircraft flap subjected to different types of loading conditions. We begin by presenting the vibrating modes of a cantilever beam to justify the exact positioning of the optical fiber on the aircraft structure. Then, the principles of operation of the sensor are explained. After that, we describe briefly the experimental programme followed to carry out the experimental tests. Next, the experimental results are presented and discussed. Finally, we summarize the main conclusions.

\section{THEORETICAL BACKGROUND}

In order to gain an insight into the real strain distribution along the flap structure and to be able to justify later (in Section IV-A) the positioning of the sensor-fiber on the aircraft flap surface, this section summarizes the theoretical aspects of the vibrating modes of an isotropic cantilever beam. The cantilever beam model, which provides a means of calculating 


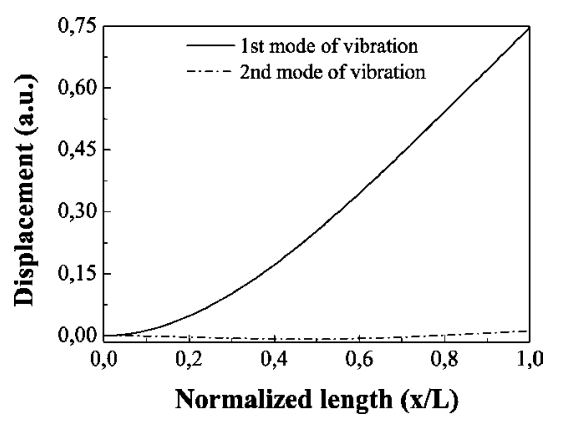

Fig. 1. Displacement caused by the first two modes of vibration of a cantilever beam as a function of the normalized beam length $x / L$ at $t=0$.

the load-carrying and deflection characteristics of beams, has been chosen for an approximated analysis of the flap rudder.

Let us consider a cantilever beam subjected to a point load $P$ at its free end. When the load is removed from the displaced beam, the beam will return to its original shape. However, inertia of the beam will cause the beam to vibrate around that initial location. Assuming that the elastic modulus $E$, the inertia $I$, and the cross-sectional area $A$ are constant along the beam length $(x)$, the equation for the free vibration is [19] and [20]

$$
E I \frac{\partial^{4} w(x, t)}{\partial x^{4}}=-\lambda_{m} \frac{\partial^{2} w(x, t)}{\partial t^{2}}
$$

where $w(x, t)$ is the deflection of the cantilever beam, $\lambda_{m}$ the linear mass density of the beam, and $-\lambda_{m} \partial^{2} w(x, t) / \partial t^{2}$ the load function used to model the vibration of the beam. The solution to this differential equation is

$$
\begin{aligned}
w_{n}(x, t)= & X_{n}(x) T_{n}(t) \\
X_{n}(x)= & \sin \left(k_{n} x\right)-\sinh \left(k_{n} x\right) \\
& -\left(\frac{\sin k_{n}+\sinh k_{n}}{\cos k_{n}+\cosh k_{n}}\right)\left[\cos \left(k_{n} x\right)-\cosh \left(k_{n} x\right)\right] \\
T_{n}(t)= & A_{n} \cos \left(\hat{\omega}_{n} t\right)
\end{aligned}
$$

where the constants $k_{n}$ and $\hat{\omega}_{n}$ are related by

$$
k_{n}^{4}=\frac{\hat{\omega}_{n}^{2} \lambda_{m}}{(E I)} .
$$

The amplitude $A_{n}$ depends on the initial position of the beam at $t=0$ in the following way:

$$
A_{n}=\frac{2}{L} \int_{0}^{L} w(x, t=0) X_{n}(x) d x .
$$

Fig. 1 shows the displacement caused by the first two modes of vibration at $t=0$. The displacement is given in arbitrary units. Notice that the third and higher order modes have not been represented, because the contribution to the overall displacement of the beam becomes smaller as the mode number increases.

The strain describes the relative deformation or change in shape and size of a material under applied forces. It can be expressed, in the case of a beam, in terms of the radius of curva-

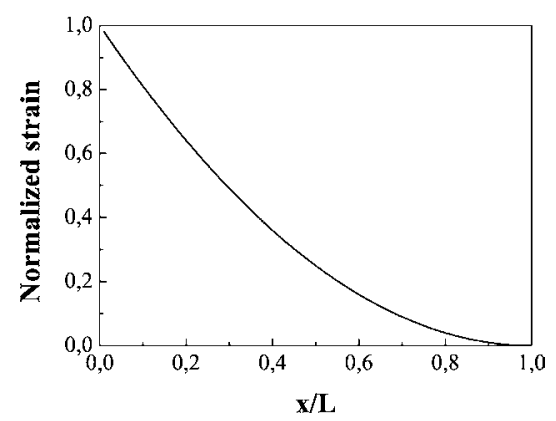

Fig. 2. Normalized strain $\bar{\epsilon}_{x}$ as a function of the normalized position along the flap $x / L$ for the first mode of vibration and at a particular instant of time. $L$ : length of the flap.

ture of the beam, $\rho$, and the distance from the neutral axis of the beam, $y$, in the following way:

$$
\epsilon_{x}=-\frac{y}{\rho} .
$$

This equation establishes that the longitudinal deformations are proportional to the curvature and change linearly with the distance $y$ from the neutral axis. For small rotations of the beam in which the curvature is small, the radius of curvature can be written as

$$
\frac{1}{\rho}=\frac{d^{2} w}{d x^{2}} .
$$

Fig. 2 shows the normalized flexural strain distribution along the flap for the first mode of vibration. The positive value of the strain represents that there is an elongation of a carbon fiber, which is located at a vertical distance $y$ and subjected to tensile load. It can be seen that the strain decreases along the length of the beam from its maximum value at $x=0$ to the tip of the flap at $x=L$.

\section{SENSOR PRINCIPLES}

Fig. 3 shows schematically the configuration of the POFbased elongation sensor. The POF is the sensitive element of the system. It has the following characteristics: a step-index refractive-index profile, a numerical aperture of 0.5 , and a core diameter of $980 \mu \mathrm{m}$. These characteristics make it multimode at the wavelength of $650 \mathrm{~nm}$. The voltage-controlled oscillator (VCO) sends a sinusoidal electronic signal with an adjustable modulation frequency $f_{m}$ to the emitter. The emitter converts the electronic signal into an optical one and transmits it to the Y-coupler. The Y-coupler splits the signal into two equal optical signals: one propagates along the measuring fiber 1 and the other one along the measuring fiber 2 . When the measuring fiber 1 elongates under stress, the light propagating along the measuring fiber 1 has to cover a longer distance and, therefore, the phase difference between both optical signals $\left(\varphi_{1}-\varphi_{2}\right)$ changes. The two photodetectors (PD1 and PD2) convert the optical signals into electrical ones, and the phase comparator $(\Delta \Phi)$ compares them in order to detect the phase shift, and it reports the corresponding voltage. The data acquisition system (A/D) from National Instruments (model NI-USB6210) offers 


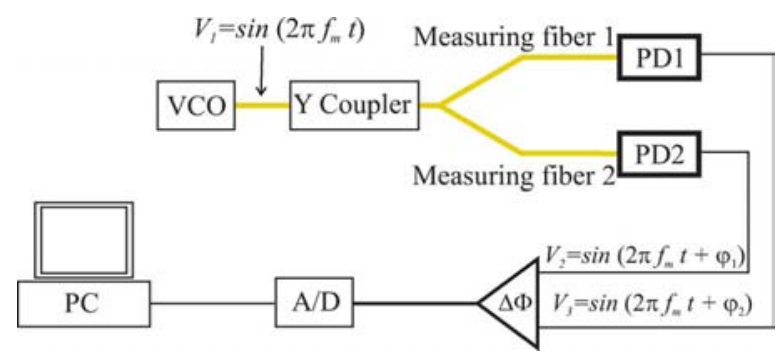

Fig. 3. Schematic representation of the sensor principle. VCO: voltage-controlled oscillator, PD1: measuring photodetector 1, PD2: measuring photodetector 2, $\Delta \Phi$ : phase comparator, $\mathrm{A} / \mathrm{D}$ : analog-to-digital converter.

up to a 16-bit resolution in the analog-to-digital conversion of the output voltage from the phase comparator, which is then displayed in the computer.

The output voltage from the phase comparator can be converted into the corresponding phase shift $\Delta \phi$ by using the following relation: $10 \mathrm{mV}$ is equivalent to $1^{\circ}$ of phase shift [21]. The corresponding elongation $\Delta l$ is calculated as [22]

$$
\Delta l=\frac{c_{0}}{n_{c o} f_{m}} \cdot \frac{1}{360^{\circ}} \cdot \Delta \phi
$$

where $c_{0}$ is the speed-of-light in vacuum, and $n_{c o}$ is the core refractive index of the fiber.

\section{EXPERIMENTAL PROGRAMME}

\section{A. Preparation and Installation of the Sensor}

The POFs were surface-bonded to a flap rudder. Different bonding materials like adhesives, glass-fiber reinforcements and a fiberglass filler were tested to fix the POF to the flap. The latter was a carbon-fiber reinforced composite with a honeycomb core and a surface of a composite material made of a resin preimpregnated with carbon-fibers (prepreg). Finally, the most suitable solution for bonding the POF to the flap surface consisted in a two-step approach with an adhesive for a first fastening of the POF to the surface and then a fiberglass filler to fix the fiber position.

Fig. 4 shows a picture of the bottom surface of the flap with the POF bonded to it. The measuring fiber 1 was fixed to the top surface of the structure, and the measuring fiber 2, instead, to the bottom surface. We used a loop-shaped configuration in both cases. When bending the flap, one of the POFs was elongated whereas the other one was compressed. This gave rise to a phase shift $\Delta \phi$ between both light signals, which produced a high voltage level at the output of the phase comparator. This voltage value, which includes the fourfold-elongation experienced by the four fiber sections (two sections on the top surface and the other two on the bottom surface), was divided by four in order to obtain the voltage corresponding to the elongation of the bent wing structure.

Regarding the fiber location on the aircraft structure, the criterion chosen to install the fiber assumes that approximately free vibrations are experienced by the structure. The free vibrations of the beam are intended to simulate real service conditions of the flap rudder. This simple criterion for the installation of the fiber gives rise to two different configuration. As shown

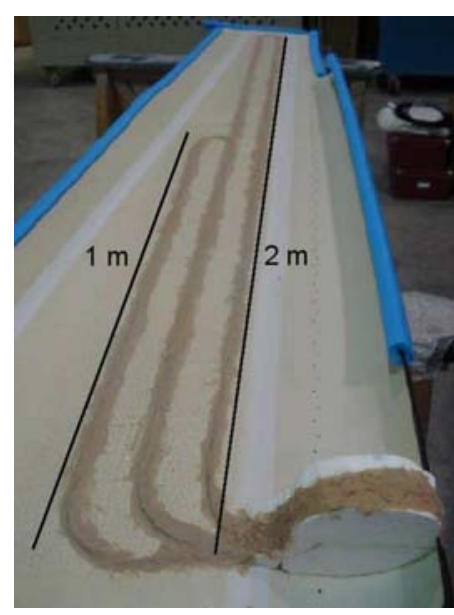

Fig. 4. Picture of the bottom surface of the aircraft flap with the POF fixed to it. The POF, in a loop-shaped configuration, has been installed with two different semilengths of $2 \mathrm{~m}$ and $1 \mathrm{~m}$.

in Section II, the first and second modes of vibration have the highest influence on the load distribution of the structure. Therefore, a convenient way of installing the fiber on the surface of the aircraft flap is to fix it longitudinally along the spatial distribution of each mode, so that two different fiber configurations will arise from it. In the first one, the loop-shaped fiber of semilength of $2 \mathrm{~m}$ extends longitudinally almost to the wing tip, following the distribution of the first mode (thus, covering half a period of the fundamental mode of vibration), whereas, in the second configuration, the $1 \mathrm{~m}$-semilength loop-shaped fiber extends along half a period of the spatial distribution of the second mode of vibration. Fig. 4 shows a picture of the disposition of both loop-shaped fibers on the bottom surface of the aircraft flap.

\section{B. Experimental Methodology}

The response of the sensor under flexural-type loading conditions was investigated in the test assembly shown in Fig. 5. The experimental setup is similar to those frequently used in typical certification tests of aircraft structures, in which the load-application velocity is not considered.

Here, one end of the flap was mounted on the interface plate attached to the framework. The load to deflect the flap tip up to $10 \mathrm{~cm}$ upwards and downwards was applied on the other end of the structure by means of an MTS 243.17 hydraulic actuator with a stroke of $508 \mathrm{~mm}$ and a nominal load of $50 \mathrm{kN}$. The maximum applied force for bending the aircraft flap to its maximum deflection value of $10 \mathrm{~cm}$ was only of $84 \mathrm{~N}$, due to the high flexibility of the wing structure. Both the interface plate and the actuator were fixed to the testing structure using clamping jaws. In order to avoid damages to the flap, the surfaces in contact with the clamping jaws were protected by $4 \mathrm{~mm}$-thick neoprene panels.

The results obtained from the fiber optical elongation sensor were compared with those obtained from a reference system. This was a 3-D camera attached to a $6 \mathrm{~m}$-high column placed near the flap. The camera detected the 3-D coordinates of the reference points, which were located directly along the installed optical fiber, and the displacement of the reference points was calculated by means of software. 


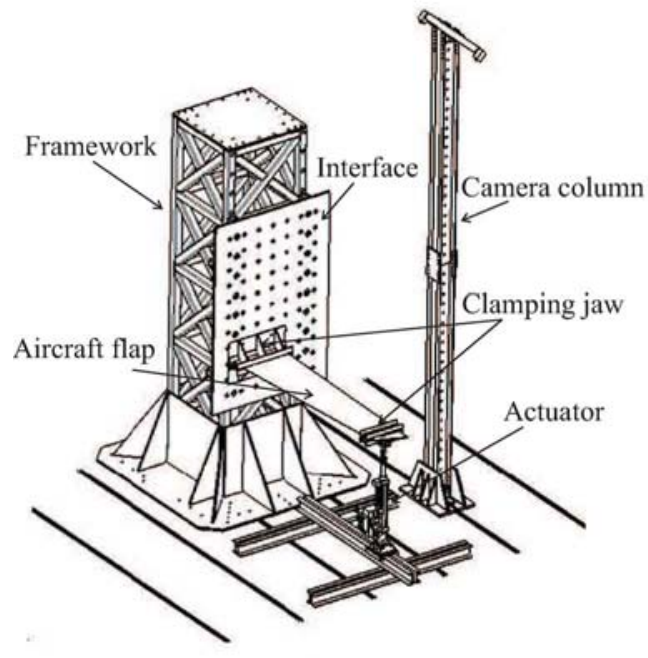

(a)

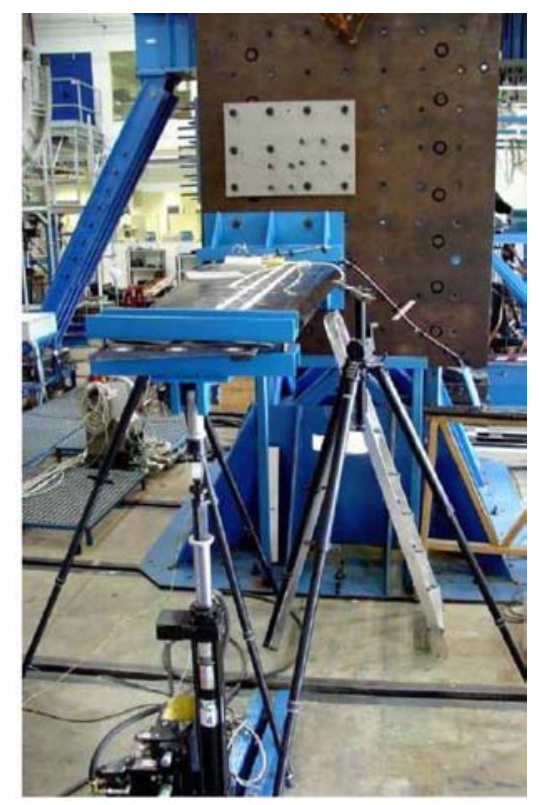

(b)

Fig. 5. Experimental setup used in the deflection tests. (a) Different components of the test assembly. (b) Picture of the test assembly.

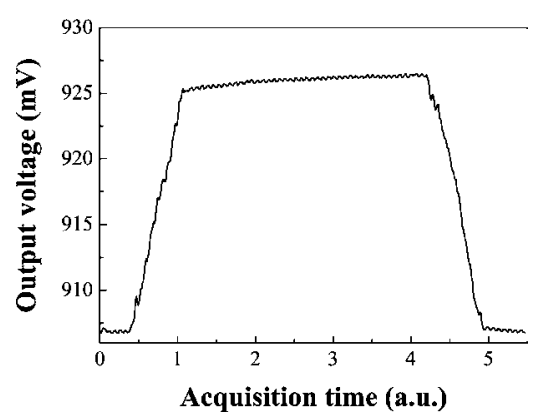

Fig. 6. Output voltage during a single-move flexural loading of the flap from the horizontal position down to the maximum flexural value of $-10 \mathrm{~cm}$ and back to the horizontal position.

In order to evaluate the functionality of the POF sensor, different tests consisting of single moves, step-type movements, and cyclical-type movements at varying velocity were carried out.

\section{RESULTS AND DisCUSSION}

Fig. 6 shows the output voltage as a result of one-cycle flexural loading of the flap from the horizontal position down to $-10 \mathrm{~cm}$ (loading process) and back to the starting position (unloading process), using the loop-shaped POF of $1 \mathrm{~m}$ of semilength. It can be seen that the output voltages at the beginning and at the end of the movement were the same and, therefore, the sensor did not exhibit any obvious sign of hysteresis at the end of the test. In what follows, unless otherwise indicated, the figures will refer to the fiber configuration of $2 \mathrm{~m}$ of semilength.

Fig. 7 plots the output voltage when the flap was deflected both upwards and downwards, starting from the horizontal position. The repeatability of the signal under flexural loading was very satisfactory. Additionally, the elongation of the aircraft

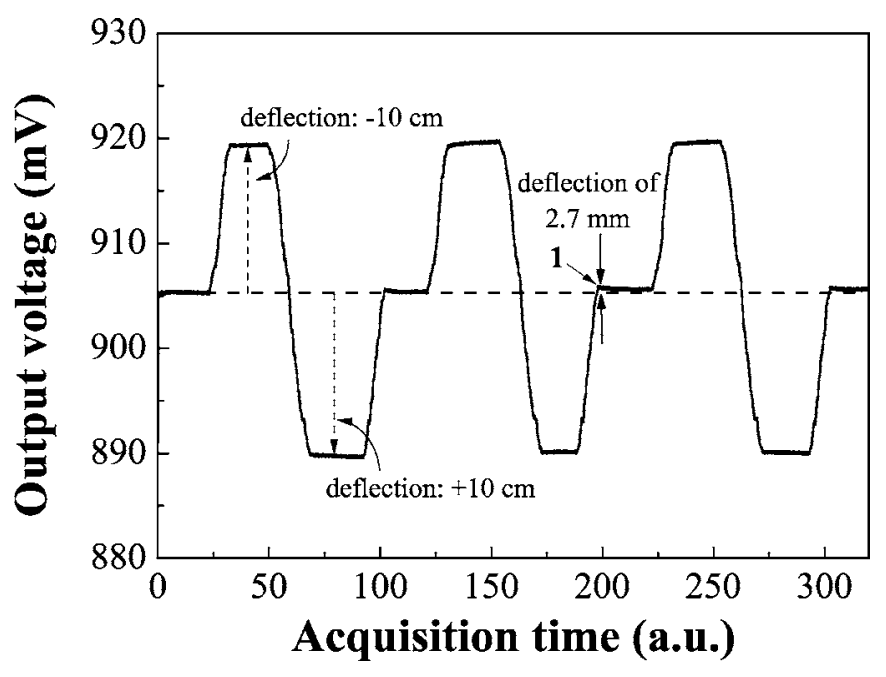

Fig. 7. Output voltage under cyclic flexural loading from $-10 \mathrm{~cm}$ to $+10 \mathrm{~cm}$.

structure for $+10 \mathrm{~cm}$ and for $-10 \mathrm{~cm}$ was nearly the same. In both measurements, the value is a distributed one. For $-10 \mathrm{~cm}$, a value of $260 \mu \mathrm{m}$ was obtained, whereas the value was $290 \mu \mathrm{m}$ for $+10 \mathrm{~cm}$. On average, the elongation measured upwards was about $30 \mu \mathrm{m}$ longer than that measured downwards. The repeatability of the sensor signal can be clearly seen from the graph, although slight variations can be detected (e.g., when we compare the output voltage levels corresponding to the horizontal position of the aircraft structure at two different cycles in Fig. 7). An uncertainty of $0.38 \mathrm{mV}$ in the measurement of the output voltage with respect to the horizontal dashed line used as reference in Fig. 7 yields an uncertainty of $2.7 \mathrm{~mm}$ in the deflection of the flap. The actuator itself can be responsible for the uncertainties obtained in the measurements.

Let us now consider the resolution of the installed POF sensor. When it is uncoupled from any structure of interest, a resolution of $5-10 \mu \mathrm{m}$ was achieved [2]. However, in order 


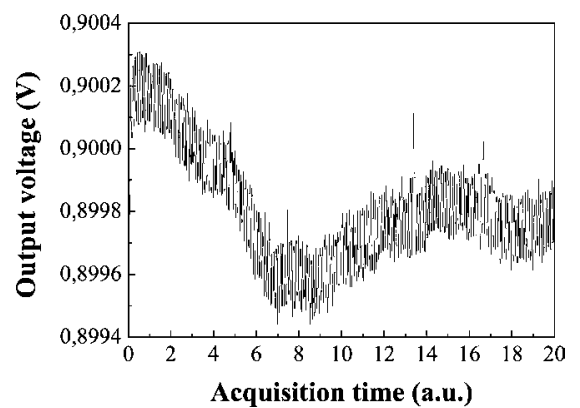

Fig. 8. Output voltage as a function of time when the actuator is at its reference position.

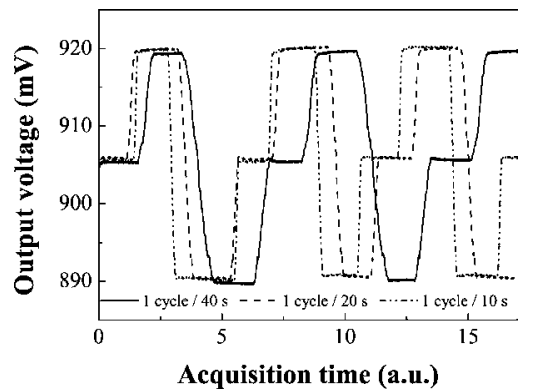

Fig. 9. Output voltage under flexural loading for three different velocities of the actuator.

to consider possible effects of the bonding process on the performance of the sensor, we have determined the resolution once the sensor was installed on the aircraft flap. Fig. 8 shows the output voltage as a function of time when the actuator is at its reference position (horizontal position of the aircraft flap). The average value is $900 \mathrm{mV}$, and the standard deviation is $3.7 \cdot 10^{-4} \mathrm{~V}$, which turns out to yield, according to (7), a resolution of $7 \mu \mathrm{m}$ for the elongation, which agrees with the value obtained for the uncoupled sensor. This resolution can be improved by considering more thoroughly several important factors such as the resolution of the actuator, or material deformation with temperature changes, but this improvement is often unnecessary in some structural monitoring applications.

The tests were also carried out at different velocities of the actuator. Fig. 9 shows the results obtained at 1 cycle/40 s, 1 cycle/20 s, and 1 cycle/10 s. It is clear that the sensor successfully monitored the loading and unloading processes of the flap, exhibiting excellent repeatability of the results in all cases. The maximum and minimum results were also independent of the velocity of the actuator.

The flexural tests were extended to investigate the effect of continuous movement on the stability of the results. In this part of the study, due to the configuration of the actuator, the movement was stopped for a short time after every cycle. Fig. 10 shows the results obtained for a displacement rate of 1 cycle/40 s. It is clear from the plot that the sensor did not undergo any observable short-term fatigue effects. The excellent stability of the maxima and minima highlights the system's repeatable response. Although not shown here, this stability can also be observed at different displacement rates of the actuator.

The third type of flexural test considered in the measurements was the step-type movement with a step-size of $1 \mathrm{~cm}$. Fig. 11 shows the output voltage obtained in the loading (up to $-10 \mathrm{~cm}$ )

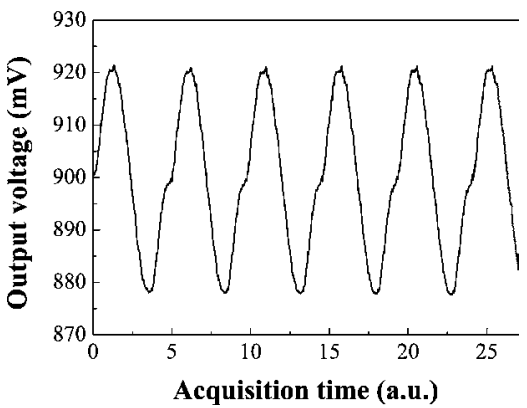

Fig. 10. Output voltage under cyclical flexural loading.

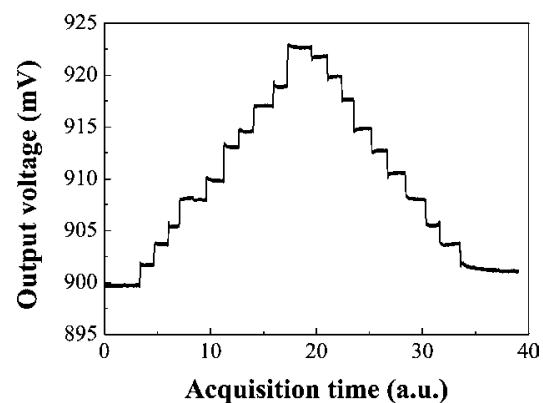

Fig. 11. Output voltage during a step-type movement of the flap. The wing moved downwards in steps of $1 \mathrm{~cm}$ from the horizontal position up to the maximum deflection value of $-10 \mathrm{~cm}$ and returned back to the horizontal position.

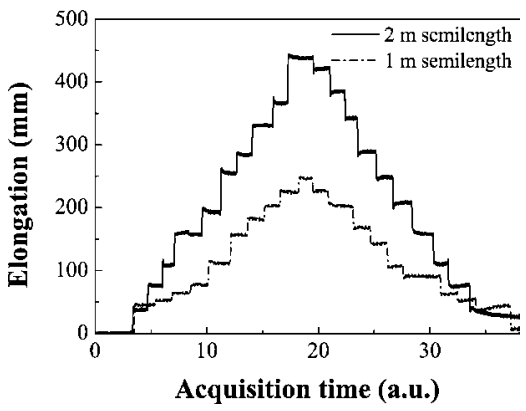

Fig. 12. Comparison of the elongations provided by the fiber optical elongation sensor corresponding to its two fiber configurations (continuous line for $2 \mathrm{~m}$ and dash dotted line for $1 \mathrm{~m}$ of semilength).

and the unloading of the flap. It can be seen that some steps show bigger increments than others. In addition, the increment of any step upwards and that of the corresponding symmetrical step downwards do not coincide with each other. As a consequence, the sensor exhibits clear signs of hysteresis at the end of the test. This can be explained by taking into account the distributed nature of the sensor, which does not record the local response at fixed points on the surface of the structure, but the overall response to the flexural loading applied to the structure. In order to clarify this point, let us consider the two configurations of the fiber optical elongation sensor, namely the $1 \mathrm{~m}$-semilength loop-shaped fiber and the $2 \mathrm{~m}$-semilength one. Fig. $12 \mathrm{com}$ pares the elongation data obtained in both cases in the step-type test. The maximum elongation provided by the fiber of $1 \mathrm{~m}$ of semilength (first configuration) was of $246 \mu \mathrm{m}$, whereas in the $2 \mathrm{~m}$-semilength fiber (second configuration) the maximum elongation value was of $438 \mu \mathrm{m}$, which is less than twice the value provided by the $1-\mathrm{m}$-configuration. This suggests that the fiber 


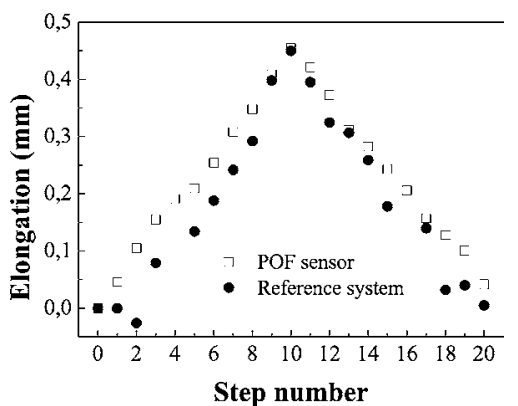

Fig. 13. Comparison of the elongations provided by the fiber optical elongation sensor and the camera-based reference system. Step-type movement with a step size of $1 \mathrm{~cm}$

optical elongation sensor detected a higher elongation along the first meter of the flap starting from the clampled end than along the second meter (nearer to the flap tip). This implies that the strain values are higher near the clampled end of the structure than near the tip, a fact well known from Euler-Bernoulli beam theory and shown graphically in Fig. 2. In addition, it is also worthy of note in Fig. 12 that the response of the sensor is more symmetrical in the case of the $1 \mathrm{~m}$-semilength fiber. This can be explained by taking into account that the fiber of $1 \mathrm{~m}$ of semilength is installed on a surface region where the fiber yields greater phase shifts due to the higher strain values, so that, for a fixed deflection value of the flap tip, the response of the sensor is better in the 1-m configuration. This argument is supported in [23], where step-type tests carried out on an aircraft structure with fiber Bragg grating-based lumped sensors have shown that the response of the sensor was better (more symmetrical and with no hysteresis) when it was installed near the clamped end than when it was installed near the tip.

In order to validate the results obtained in the step-type tests with the fiber optical elongation sensor, we have compared them with those provided by the reference system. Fig. 13 shows the elongations provided by both the fiber optical elongation sensor and the camera-based system in the step-type flexural test shown in Fig. 11. The reader is cautioned that the camerabased system is usually used to measure smaller deformations such as those occurring in vibration analyses. For this reason, in the case of stronger deformations, as in our case, the measurements are not as accurate as those obtained in small deformation analyses. This can give rise to anomalous elongation values or even missing elongation values. Although two data points (data corresponding to step numbers 4 and 16 in Fig. 13) are missing, and although some other points (data corresponding to step numbers 2 and 18) are out of range in the measurement of the camera-based system shown in Fig. 13, the results are relatively good and come to confirm the validity of the results obtained with the fiber optical elongation sensor, and also the better peformance of the fiber-based sensor in comparison with the camera-based system. Both sensors detect a maximum elongation of $450 \mu \mathrm{m}$ for a deflection of the wing of $+10 \mathrm{~cm}$ (step number 10).

\section{CONCLUSION}

The response of a POF-based elongation sensor surface-bonded to an aircraft flap has been investigated. The tests conducted on the flap have demonstrated the potential of the sensor for monitoring the elongation of the aircraft structure. The high degree of repeatability and lack of hysteresis of the sensor signal observed in single movements and cyclical-type movements at different velocities highlight its potential for use in different operating conditions. In addition, the comparison (when possible) of the POF sensor and the camera-based used for validating the response of the POF sensor have demonstrated the superiority of the fiber optical strain sensor.

\section{REFERENCES}

[1] C. Michie, B. Culshaw, G. Thursby, W. Jin, and M. Konstantaki, “Optical sensors for temperature and strain measurement," in Proc. Smart Sensing, Process., Instrum., Smart Structures, Mater., 1996, vol. 2718, pp. 134-146.

[2] M. Luber, H. Poisel, S. Loquai, C. Neuner, A. Bachmann, O. Ziemann, and E. Hartl, "POF strain sensor using phase measurement techniques," in Proc. 16th Int. Conf. Plastic Opt. Fibers and Appl.-POF'07, Tourin, Italy, Sep. 2007, pp. 29-32.

[3] B. D. J. Deboux, E. Lewis, P. J. Scully, and R. Edwards, "A novel technique for optical fiber $\mathrm{pH}$ sensing based on methylene blue absorption," J. Lightw. Technol., vol. 13, pp. 1407-1414, Jul. 1995.

[4] Y. Gu, Z. Qian, B. Tao, and G. Wang, "A new fiber optic sensor for detecting in situ the concentration of pharmaceuticals in blood," Sens. Actuators B, vol. 66, pp. 197-199, 2000.

[5] S. Melle, K. Liu, and R. M. Measures, "Strain sensing using a fibre optic Bragg grating," Proc. SPIE, vol. 1588, pp. 255-263, 1991.

[6] H. J. Patrick, C. C. Chang, and S. T. Vohra, "Long period fibre gratings for structural bend sensing," Electron. Lett., vol. 34, pp. 1773-1775, 1998.

[7] S. P. Christmas, D. A. Jackson, P. J. Henderson, L. Zhang, I. Bennion, T. Dalton, P. Butler, M. Whelan, and R. Kenny, "High-resolution vibration measurements using wavelength-demultiplexed fibre Fabry-Perot sensors," Meas. Sci. Technol., vol. 12, pp. 901-905, 2001.

[8] Y. J. Rao, D. J. Webb, D. A. Jackson, L. Zhang, and I. Bennion, "Infibre Bragg grating temperature sensor system for medical applications," J. Lightw. Technol., vol. 15, pp. 779-785, 1997.

[9] K. S. C. Kuang, R. Kenny, M. Whelan, W. J. Cantwell, and P. R. Chalker, "Residual strain measurement and impact response of optical fibre Bragg grating sensors in fibre metal laminates," Smart Mater. Struct., vol. 10, pp. 338-346, 2001.

[10] S. Kitade, T. Fukuda, and K. Osaka, "Fiber optic method for detection of impact induced damage in composite laminates," J. Soc. Mater. Sci., vol. 44, pp. 1196-1200, 1995.

[11] N. Takeda, T. Kosaka, and T. Ichiyama, "Detection of transverse cracks by embedded plastic optical fiber in FRP laminates," Proc. SPIE, vol. 3670, pp. 248-255, 1999.

[12] R. Maaskant, A. T. Alavie, R. M. Measure, G. Tadros, S. H. Rizkalla, and A. Guha-Thakurta, "Fiber-optic Bragg grating sensors for bridge monitoring," Cement Concrete Components, vol. 19, pp. 21-23, 1997.

[13] M. A. Davis, D. G. Bellemore, and A. D. Kersey, "Distributed fiber Bragg grating strain sensing in reinforced concrete structural components," Cement Concrete Components, vol. 19, pp. 45-57, 1997.

[14] R. A. Badcock and G. F. Fernando, "An intensity-based optical fibre sensor for fatigue damage detection in advanced fibre-reinforced composites," Smart Mater. Struct., vol. 4, pp. 223-230, 1995.

[15] D. C. Lee, J. J. Lee, I. B. Kwon, and D. C. Seo, "Monitoring of fatigue damage of composite structures by using embedded intensity-based optical fiber sensors," Smart Mater. Struct., vol. 10, pp. 285-292, 2001.

[16] S. Kiesel, K. Peters, T. Hassan, and M. Kowalsky, "Behavior of intrinsic polymer optical fiber sensor for large-strain applications," Measure. Sci. Technol., vol. 18, pp. 3144-3154, Sep. 2007.

[17] S. Kiesel, P. V. Vickle, K. Peters, O. Abdi, T. Hassan, and M. Kowalsky, "Polymer optical fiber sensors for the civil infrastructure," SPIE Smart Materials and Structures, vol. 6174, 2006.

[18] K. S. C. Kuang, W. J. Cantwell, and P. J. Scully, "An evaluation of a novel plastic optical fibre sensor for axial strain and bend measurements," Measure. Sci. Technol., vol. 13, pp. 1523-1534, 2002.

[19] D. J. Inman, Engineeering Vibration. Englewood Cliffs, NJ: Prentice Hall, 1994.

[20] E. Voltera and E. C. Zachmanoglou, Dynamics of Vibrations. Columbus, OH: Charles E. Merrill Books, 1965.

[21] "Data sheet LF-2.7 GHz RF/IF gain and phase detector AD8302," Analog Devices, Inc., 2002. [Online]. Available: http://www. analog.com

[22] C. Neuner, "Faseroptischer Längensensor Basierend auf Laufzeitmessung," M.S. thesis, Georg Simon Ohm Univ. Appl. Sci. Nuremberg, Nuremberg, Germany, 2007. 
[23] J. Gómez, J. Zubia, G. Aranguren, G. Durana, J. A. Illaro, I. Sáez, M. Kirchhof, H. Poisel, and E. Hartl, "Comparing polymer optical fiber (POF), fiber Bragg gratings and traditional strain gauge for aircraft structural health monitoring," in Proc. Avionics, Fiber-Optics Photon. Technol. Conf. (AVFOP), San Diego, CA, Sep. 2008, pp. 51-52.

Gaizka Durana received the B.Sc. degree in solid-state physics in 1999 and the Ph.D. degree in engineering in 2008, both from the University of the Basque Country, Bilbao, Spain. His Ph.D. work focused on the experimental and numerical analysis of fundamental aspects of light propagation in multimode optical fibers.

His current research interests include light propagation properties in multicore polymer optical fibers and active polymer optical fibers for amplification purposes.

Dr. Durana received a European acknowledgement of his Ph.D. degree Doctor Europeus in 2008.

Marlene Kirchhof received the S.B. degree in precision engineering from the University of Applied Sciences, Nuremberg, Germany, in 2008.

Since 2008, she has been a Construction Engineer at Magnet Schultz GmbH, Memmingen, Germany.

Michael Luber received the S.B. degree in engineering from the University of Applied Sciences, Nuremberg, Germany.

He is currently a Staff Member at the Polymer Optical Fiber Application Center (POF-AC), Nuremberg.

Idurre Sáez de Ocáriz received the M.Sc. degree in solid-state physics and the Ph.D. degree in physics from the University of the Basque Country, Bilbao, Spain, in 1995 and 2001, respectively. Her Ph.D. work focused on the optical properties (laser spectroscopy) of $\operatorname{Pr} 3+$ in crystals and glasses. She received the Postgraduate in Manangement and Organization of Research and Innovation from the Polytechnic University of Madrid (UPM), Madrid, Spain, in 2005.

She is currently Head of R\&D at CTA, Aeronautical Technological Center, Vitoria, Spain.
Hans Poisel (M'91) received the M.Sc. degree in physics from the Technical University Munich, Munich, Germany, in 1977 and the Ph.D. degree from the Faculty of Electrophysics, Technical University Munich, in 1983.

Since 1983, he was working for IABG, Ottobrunn, on simulation of radar countermeasures. In 1985, he joined MBB in Ottobrunn/Munich, active in development of fiber-optic sensors, leading the fiber-optic gyro project and, furthermore, coordinating all fiber-optic activities of the company as a whole. Since 1991, he has been a Professor of Technical Optics and Optical Communication at the University of Applied Sciences, Nuremberg, Germany. He published more than 50 contributions devoted to optical properties of plastic optical fibers and their applications in international conferences and journals and holds more than 50 patents in the area of fiber-optics. Currently, he is a member of the International Committee for Plastic Optical Fibers (ICPOF) and Director of Polymer Optical Fiber Application Center (POF-AC), Nuremberg.

Prof. Poisel is a member of OSA, VDE, and DPG.

Joseba Zubia received the M.Sc. degree in solid-state physics and the Ph.D. degree in physics from the University of the Basque Country, Bilbao, Spain, in 1988 and 1993, respectively. His Ph.D. work focused on the optical properties of ferroelectric liquid crystals.

$\mathrm{He}$ is currently a Full Professor with the Department of Electronics and Telecommunications, School of Engineering of Bilbao, University of the Basque Country. He has more than 12 years of experience doing basic research in the field of polymer optical fibers and is currently involved in research projects in collaboration with universities and companies from Spain and other countries in the field of polymer optical fibers, fiber-optic sensors, and liquid crystals.

Prof. Zubia was a recipient of a Special Award for Best Thesis in 1995.

Carmen Vázquez received the M.Sc. degree in physics in 1991 from Complutense University of Madrid, Madrid, Spain, and the Ph.D. degree in engineering in 1995 from Polytechnic University of Madrid (UPM), Madrid.

She is currently working as an Associate Professor at Carlos III University of Madrid. She has published more than 140 papers in journals and conferences. Her current research interests include ring resonators, plastic optical fibers, broadband access networks, and LC and integrated optic devices and their applications to optical communications and sensor networks. 